\title{
Synthesis and photochemical reactions of iron(II) doped copper ferrites as heterogeneous catalysts
}

\author{
Asfandyar Khan ${ }^{1}$, Zsolt Valicsek ${ }^{1}$, Ottó Horváth ${ }^{1}$ \\ ${ }^{1}$ Department of General and Inorganic Chemistry, Faculty of Engineering, University of Pannonia \\ Veszprém, Hungary \\ asfandyar.khan@almos.uni-pannon.hu
}

\section{Extended Abstract}

Synthetic dyes and pigments have been widely used for aesthetic purpose, mainly in textile dyeing and printing, food, ink, papermaking, cosmetics and pharmaceutical industries. Synthetic dyes are preferred over natural counterparts because of its brilliant colours and reasonable prices. Among these dyes methylene blue (MB) is considered as hazardous to many living organisms including aquatic lives. Researchers have investigated many treatment methods such as adsorption, Fenton process, biological oxidation, sonochemical and photochemical oxidation to eradicate MB from aqueous medium[1].

Recently heterogeneous ferrite NPs have attracted the researcher's attention in wastewater treatment process, in the photocatalytic degradation of various hazardous compounds [2].

This research work reported the synthesis of magnetic iron(II) doped copper ferrite nanoparticles (NPs) by simple coprecipitation method.

The NPs were synthesized with change in composition of copper and iron(II) given as, $\mathrm{Cu}_{(\mathrm{x})}^{\mathrm{II}} \mathrm{Fe}^{\mathrm{II}}(1-\mathrm{x}) \mathrm{Fe}^{\mathrm{III}}{ }_{2} \mathrm{O}_{4}($ where $\mathrm{x}=$ $0.0,0.2,0.4,0.6,0.8,1.0)$. The samples were characterized by Raman spectroscopy, XRD, SEM, TEM-EDS and particle size analysis. The synthesized NPs were evaluated for photocatalytic activity using methylene blue (MB as the model hazardous dye) in the presence of hydrogen peroxide (as the reagent of Fenton type system). The photocatalytic activities were determined due to the continuously measured UV-visible spectra during the irradiation of MB. The $\mathrm{Cu}_{(0.4)} \mathrm{Fe}_{(0.6)} \mathrm{Fe}^{\mathrm{IIII}}{ }_{2} \mathrm{O}_{4}$ NPs were observed to be the best among the synthesized NPs. The effects of $\mathrm{pH}$, concentration of NPs and $\mathrm{H}_{2} \mathrm{O}_{2}$ were investigated on dye degradation; hence, the optimized circumstances were determined for the highest degradation efficiency. It can be concluded based on the photocatalytic results that iron(II) doped copper ferrites will be a new catalyst in wastewater treatment.

\section{Acknowledgement}

This work was supported by the Széchenyi 2020 under the GINOP-2.3.2-15-2016-00016 and the EFOP-3.6.1-16-201600015.

\section{References}

[1] V. S. Kirankumar and S. Sumathi, "Photocatalytic and antibacterial activity of bismuth and copper co-doped cobalt ferrite nanoparticles," Journal of Materials Science: Materials in Electronics, vol. 29, pp. 8738-8746, 2018.

[2] Z. Zhu, X. Li, Q. Zhao, Y. Li, C. Sun, and Y. Cao, "Photocatalytic performances and activities of Ag-doped CuFe ${ }_{2} \mathrm{O}_{4}$ nanoparticles," Materials Research Bulletin, vol. 48, pp. 2927-2932, 2013. 